\title{
Portable hybrid power storage system
}

\begin{abstract}
The shortage of electrical power for electronic devices like mobile, laptop, instant light or fan in remote areas where utility power is not available. Usually, ultimate power supply (UPS) with a generator is used to fulfill the electrical power requirement in absence of utility. UPS can provide power in AC only and cannot carry from one place to another due to its heavy weight and fixed installation at particular place. In this research, a portable device which, can be carried to any place and can provide continues power in both AC and DC. This portable device having a new AC/DC converter circuit and used together with battery and referred to as a portable hybrid power storage system. This proposed system has the advantage of enhancement in the range of battery charging current control. It's an environment-friendly system. It can provide six hours of power in both AC and DC form up to 500W electronic appliances such as lamps, fans or mobile charges. It has multiple ports that can take input from solar, generator or utility. Moreover, the optimal battery sizing is also done for its optimal operation.
\end{abstract}

Keyword: Portable hybrid power storage system (PHPSS) 